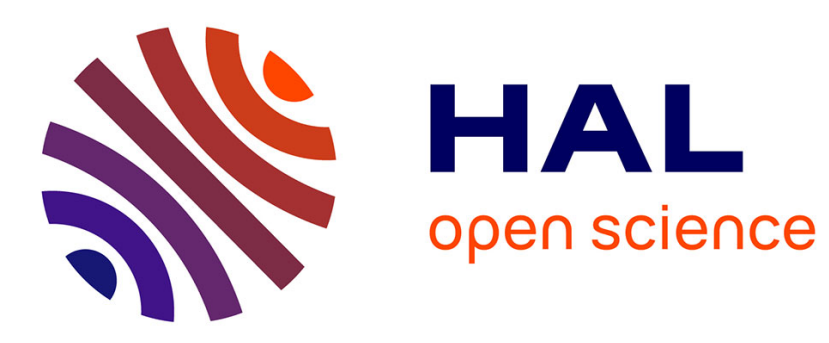

\title{
L'image médicale à l'épreuve de la psychanalyse Paul-Laurent Assoun
}

\section{To cite this version:}

Paul-Laurent Assoun. L'image médicale à l'épreuve de la psychanalyse: Le fantasme iconographique. Recherches en psychanalyse, 2009, Les effets psychiques de l'image en médecine: regards croisés sur l'imagerie médicale, 8, 10.3917/rep.008.0182 . hal-01501937

\section{HAL Id: hal-01501937 https://hal.science/hal-01501937}

Submitted on 4 Apr 2017

HAL is a multi-disciplinary open access archive for the deposit and dissemination of scientific research documents, whether they are published or not. The documents may come from teaching and research institutions in France or abroad, or from public or private research centers.
L'archive ouverte pluridisciplinaire HAL, est destinée au dépôt et à la diffusion de documents scientifiques de niveau recherche, publiés ou non, émanant des établissements d'enseignement et de recherche français ou étrangers, des laboratoires publics ou privés. 


\section{L'IMAGE MÉDICALE À L'ÉPREUVE DE LA PSYCHANALYSE Le fantasme iconographique \\ Paul-Laurent Assoun}

Association Recherches en psychanalyse | « Recherches en psychanalyse »

2009/2 n 8 | pages 182 à 189

ISSN 1767-5448

Article disponible en ligne à l'adresse :

http://www.cairn.info/revue-recherches-en-psychanalyse-2009-2-page-182.htm

\section{Pour citer cet article :}

Paul-Laurent Assoun, «L'image médicale à l'épreuve de la psychanalyse. Le fantasme iconographique », Recherches en psychanalyse 2009/2 ( ${ }^{\circ}$ 8), p. 182-189. DOI 10.3917/rep.008.0182

Distribution électronique Cairn.info pour Association Recherches en psychanalyse.

(C) Association Recherches en psychanalyse. Tous droits réservés pour tous pays.

La reproduction ou représentation de cet article, notamment par photocopie, n'est autorisée que dans les limites des conditions générales d'utilisation du site ou, le cas échéant, des conditions générales de la licence souscrite par votre établissement. Toute autre reproduction ou représentation, en tout ou partie, sous quelque forme et de quelque manière que ce soit, est interdite sauf accord préalable et écrit de l'éditeur, en dehors des cas prévus par la législation en vigueur en France. Il est précisé que son stockage dans une base de données est également interdit. 


\section{Recherches en Psychanalyse - Research in Psychoanalysis}

8| 2009 - Les effets psychiques de l'image en médecine : regards croisés sur l'imagerie médicale The Psychical Effects of the Image in Medicine: Contrasting Perspectives on Medical Imagery

\section{L'image médicale à l'épreuve de la psychanalyse}

Le fantasme iconographique

Medical Imagery Put to the Test of Psychoanalysis

The Iconographic Fantasy

Paul-Laurent Assoun

\section{Résumé :}

La présente contribution s'inscrit dans une recherche croisée sur la clinique psychanalytique du corps (Cf. P.-L. Assoun, Corps et symptôme, Leçons de psychanalyse, 3e éd., 2008) et sur le lien entre corps, science et lien social (Cf. P.-L. Assoun, Freud et les sciences sociales, 2e éd., Armand Colin, 2008) développée dans la spécialité du Master recherche Université Paris VII Denis Diderot dont nous sommes responsable, "Cliniques du corps et anthropologie psychanalytique ». Nous renvoyons dans les notes aux contributions susceptibles d'expliciter les dimensions évoquées ici.

\section{Abstract :}

This article derives from a joint research project on the psychoanalytic clinic of the body (Cf. P.-L. Assoun, Corps et symptôme, Leçons de psychanalyse, 3e éd., 2008) and the connections between the body, science and the social bond (Cf. P.-L. Assoun, Freud et les sciences sociales, 2e éd., Armand Colin, 2008), developed as part of the Research Master's program "Clinic of the body and psychoanalytic anthropology" we direct at the University Paris 7 Diderot. We refer the reader to the footnotes for works developing some of the dimensions mentioned here.

Mots-clefs : imagerie médicale, psychanalyse, anthropologie psychanalytique

Keywords : medical imaging, psychoanalysis, psychoanalytic anthropology

\section{Plan :}

L'impératif scopique et la clinique

L'icono-graphique

Écriture de l’image, écriture par l'image : «l'organologique »

L'« imagier médical » ou le vouloir-voir

L'inconscient de l'image : (sa)voir et "ça voir "

Le corps en miettes

Photographier le délire

Le fantasme froid de la science : l'avenir d'une illusion

L'œil artificiel ou le fantasme de la science

La " schize » de l'œil et du regard

L'autorité aveugle de l'image : le regard clinique 
Un « jouet magnifique », mais qui « contraint l'œil à endosser un uniforme ", disait Franz Kafka du cinéma. ${ }^{1}$ Doit-on aborder de même, d'un œil vigilant et critique, les techniques de l'image qui se trouvent à présent au cœur de la médecine ? Du moins s'agit-il ici de déterminer ce que la psychanalyse peut dire en propre sur ce que l'on nomme "imagerie médicale».

Celle-ci nous confronte aux "techniques de pointe " de l'investigation médicale. Dimension particulière et privilégiée de la médecine que I'on appelle scientifique et qui s'avère en cette occasion proprement technologique. Secteur de pointe de l'innovation pendant le dernier quart de siècle qui inaugure l'ère imagière du savoir médical. Révolution de fait de la médecine scientifique, qui ouvre son ère technologique, l'ère "imagière ", au-delà de l'inventaire des accessoires, mérite sa théorie.

Cette technologie se présente comme destinée à améliorer, voire fonder le diagnostic, facilitant le traitement pour de nombreuses affections. II se peut même, qu'à terme, elle redéfinisse la notion même de diagnostic, réduit à une "monstration " qui prétend y trouver une forme d'accomplissement. L'enjeu en est tangible, soit l'identification spatialisée précise de la lésion rendant possible le recours éventuel à l'acte chirurgical, en en aiguisant la stratégie par rapport à la géographie corporelle. Ainsi semble-t-elle promettre l'accès le plus direct de visu - au mystère même de l'organe.

Cela, c'est ce que dit en substance le discours médical. Comment la psychanalyse, attentive à occuper sa position dans la condition contemporaine de la science, depuis la position du sujet inconscient, y inscrit-elle sa lecture ?

\section{L’impératif scopique et la clinique}

Il convient d'abord de prendre le recul nécessaire afin de centrer ce moment " imagier » dans la genèse de la médecine scientifique.

Rappelons-en la logique: dans le paradigme inaugurateur de la clinique moderne, anatomo- pathologique, le corps se découpe selon l'espace de visibilité - tournant consommé aux XVIIle-XIXe siècle avec "la naissance de la clinique " dont Michel Foucault dressa le portrait archéologique. Le corps est tel, en sa vérité scientifique, qu'il se donne à voir. On ne croit que ce que l'on voit : cet adage est devenu celui de la science, au point que la vision s'impose comme le mode privilégié de la créance médicale. Tel est l'impératif scopique inaugurateur de la médecine scientifique, symbolisé par la fameuse interpellation à laquelle se confronte désormais le patient: "où ?", impératif de localisation proféré comme " où avez-vous mal ?"

Que l'on se reporte à l'autre bout de l'évolution, et l'on voit cette exigence radicalisée et " internalisée » : c'est le dedans de l'organe qui doit être soumis à cet impératif de visibilité, au point de s'imposer comme " photographie » du symptôme. Au-delà de quelque grossier réalisme du "voir", se développe une technologie du regard des plus sophistiquées. Regard armé - " jusqu'aux dents ", en quelque sorte - de la technologie. La moindre des choses est de se demander ce que signifie - et donc implique - cette mutation de la clinique médicale quant au statut du sujet et du symptôme.

\section{L’icono-graphique}

Nous commencerons par balayer nous-même, d'un regard de survol, l'arsenal de techniques référées à l'imagerie médicale, afin de dégager le principe de son action, soit l'imagerie médicale à l'assaut du " dedans » corporel.

Tout part de la radiographie qui utilise les rayons $X$ mis à jour par Röntgen (1895) qui ont la capacité de traverser le corps et de le soumettre à la découpe en l' « impressionnant ". $\mathrm{Ce}$ que l'on obtient alors, c'est un film radiographique, plus ou moins noirci en fonction de l'organe traversé. Ce qui se dessine a pourtant déjà structure de fantasme: la "radio » ressemble à une ombre chinoise, où 
les os apparaissent en blanc et les structures moins denses (comme les poumons) en noir. Technique rigoureuse du clair-obscur, ainsi haussé au rang d'art scientifique. L'art du clairobscur sert d'organon à la science, éprise de "clair " et de "distinct ", en ce réseau d'opacification que désigne le corps.

Mais voici qu'interviennent les " ultra-sons » et les techniques d'exploration afférentes, telle l'échographie. Le faisceau d'ultrasons pénètre dans la zone du corps à explorer au moyen d'une sonde. C'est la réflexion de ces ondes selon leur degré de puissance- qui révèle comme magiquement la nature des tissus. Le traitement de ces "échos" permet une visualisation des organes observés. Étonnant croisement entre l'auditif et le scopique: à l'adage "l'œil écoute", se substitue "le son voit ", plus précisément : I'ultrason fait voir...

Avec le scanner, la radiologie des rayons $X$ déployant dans le tube exécutant son mouvement rotatif autour du patient, le film se trouve remplacé par des capteurs. Cela permet d'explorer, par "rotation continue ", le volume corporel - en l'espace de quelques secondes. Raccourcissement temporel saisissant par la technique dite tomographique (" en coupes »). C'est dans le traitement et par la reconstruction de ces données que paraissent émerger les images des structures. Tant la structure ici semble rejoindre l'image, l'image décalquant littéralement et matériellement la structure.

Cela culmine dans l'imagerie par résonance magnétique. Cette fois, c'est au moyen d'un aimant - de taille considérable - que se trouve analysée la réaction des différents tissus du corps à ces champs magnétiques. La zone étudiée, restituée en deux ou trois dimensions, permet de visualiser des détails invisibles sur les radiographies standards, échographie ou scanner, d'autant que les données recueillies sont ensuite traitées informatiquement - sauf à ce que le délai d'obtention des résultats soit rallongé.

Reste la scintigraphie, examen de médecine nucléaire qui, par injection dans une veine d'un produit légèrement radioactif, permet de photographier l'organe. C'est le temps de fixation progressive du produit qui permet de cerner l'organe à observer. L'appareil, appelé gamma caméra, capte les signaux émis par le produit, fixé de façon différentielle dans le corps. Le sang, en courant dans les veines, y répercute la puissance de l'image.

Écriture de l'image, écriture par l'image : "l'organologique »

De ce tableau littéralement impressionnant, ressort une écriture (graphie) endo-scopique. Corps bombardé par un arsenal de techniques destiné à en extraire sa vérité scopique, par un montage original dont on peut dégager la syntaxe.

Ce qui en ressort, c'est que le regard s'écrit. $C^{\prime}$ est ce qui confère son aspect magique à cette technologie scientifique, imprimant sur le spectateur, par ses effets, ce sceau de " prodige ». L'effet " lanterne magique " prend là ses titres de noblesse scientifique, tant la science, qui rompt de principe avec la magie, semble y ramener.

Nous sommes dans une logique de la découpe ana-lytique. C'est la structure en creux que dessine le regard appareillé par la science médicale : I'introduction de l'instrument endoscopique dans le conduit ou la cavité de l'organisme permet d'en produire un eidôlon.

Corrélativement, apparaît une sorte de sémiotique : sur le corps balayé par l'imagerie, s'allument des "signaux» qui le rendent décodable à souhait.

Enfin cette image est à la fois matérielle et virtuelle, puisque c'est de la reconstruction informatique que jaillit l'image in fine, après traitement physique selon les diverses modalités mentionnées. Le plus significatif est que le réalisme des volumes se convertit en une désignation d'autant plus efficacement localisatrice que virtualisée.

C'est l'organe qui est visualisé in vivo et de I'intérieur. Le regard médical ne fait plus qu'épouser les formes du vivant, il le pénètre. 
Progression logique de l'érotique médicale comme érotique d'organes! Cela relève en fait d'une organologie qui est au fond très peu érotique, car le rayonnement (sous ses formes physiques variées) semble traverser le corps sans y toucher, de façon la moins avasive qui soit. La production du regard semble destinée à faire l'économie de l'effusion de sang. Plus question de faire saigner inutilement le corps... sauf à ce que le corps soit secrètement blessé par le regard même et que l'image coule en quelque sorte dans les veines du sujet...

\section{L’« imagier médical » ou le vouloir-voir}

La question que peut articuler la psychanalyse, au-delà de la fascination du spectacle, c'est: que veut "l'imagier médical " ? Pas seulement mieux voir, comme il le dit, mais voir. Voir quoi ? On est supposé y avoir répondu: visualiser l'événement local avec son germe de pathologie. Ce qui se révèle du "scanner » à la lueur de la clinique analytique, c'est qu'il vise, au-delà de l'objet, le "voir ", ici absolu substantivé. En cette passion placide de surprendre le corps, se détache l'objet du regard. ${ }^{2}$ Cette passion de voir l'objet en détail(s) tend à ériger le " regarder » en idéal.

Voir devient bien alors un absolu, intransitif en quelque sorte. Nous ne nous engageons pas pour autant dans quelque psychanalyse du " savant " ou du médecin, mais des conditions inconscientes du dispositif dans lequel est pris lui-même le "sachant-voyant " comme instance. Nous sommes pris dans cette scopicité foncière de la science. Mouvement qui va du savoir au " voir ça »... " Voyez-vous ça ? ", voilà la question qui ordonne la recherche, ce qui s'inscrit en retour en effet épatant : « Voyez-vous ça ! "

\section{L'inconscient de l'image : (sa)voir et «ça voir »}

Nous pouvons indiquer les coordonnées métapsychologiques de ce mouvement. Cela nous permettra de mettre à jour ce que dissimule cette venue de l'image sur le devant de la scène.

Tout d'abord se démontre la radicalisation d'un mouvement inhérent à la pulsion. La " pulsion scopique " est repérée par Freud comme inhérente au savoir - avec son complément d'emprise. ${ }^{3}$

Il y a une scopicité de la pulsion, qui va du plaisir de contempler (Schaulust) à la pulsion de voir (Schautrieb), polarisée autour du « voir ça » ou "ça-voir".

Freud parle d'une "étape préliminaire de la pulsion de voir, dans laquelle le plaisir de voir a le corps propre pour objet ", dont il dit qu' " elle appartient au narcissisme, qu'elle est une formation narcissique. À partir d'elle se développe la pulsion de voir active... ". ${ }^{4}$ Par un mouvement étonnant, cette pulsion de voir œuvrant au cœur de l'activité scientifique semble ramener à sa source physique.

On retrouve la dimension de l'image dans les formations inconscientes. La prise en compte de la figurabilité (Rücksicht auf Darstellbarkeit), cet élément essentiel du rêve, en révèle la pression. Le terme fait allusion à des "égards " ou prise en considération de la représentation, là où la perversion fait de la Darstellbarkeit un impératif fonctionnel.

Cela renvoie à l'expérience de satisfaction, l'objet de la satisfaction primitive étant de l'ordre de l'hallucination. Voir ici et maintenant entoure une aura d'invisibilité, tout en renvoyant à ce quelque chose d'originaire localisable dans la "chose » hallucinée (dans l'expérience de satisfaction).

\section{Le corps en miettes}

L'idéal spéculaire de la médecine ramène curieusement à l'avant unité spéculaire: la médecine investigue des "bouts de corps ". Elle gère scrupuleusement la dés-articulation savante du corps. Comme si, le temps de la maladie et de l'examen médical, se trouvait remis en morceaux, pour "le bien » du sujet, I'unité péniblement conquise entre 6 et 18 mois! " Pour son bien ", car l'idée est que, plus 
nous serons bien et complètement vus, plus notre corps sera transparent, plus il saura se défendre comme les opacités du mal. On trouve là la hantise de la tumeur. Aussi, le sujet, dès qu'il soupçonne une affection, offre-t-il avec empressement son corps au découpage salutaire qui devrait permettre de dépister la cause du malaise. Même l'hypocondriaque, ce champion de l'opacité, se mettant à la page, paie son tribut à ce test de transparence. On peut tenter de venir à bout de sa transe spéculaire au moyen de l'évidence de l'image: voire, l'hypocondriaque, " malade imaginaire ", peut renchérir en exigeant toujours plus de transparence, afin de vaincre cette voix des distorsions narcissiques qui met au défi la créance médicale...

Qu'il y ait là ignorance du sujet et déni du corps inconscient - en ses dimensions pulsionnelles, narcissiques et mortifères telles que les révèle une métapsychologie de la corporéité ${ }^{5}$ - va de soi, mais ouvre une question : pourquoi ce déni trouve-t-il sa scientificité dans l'ordre de l'image ? Là prend forme un diagnostic: cette passion de l'image procède du forçage scopique, corrélatif du déni dont se soutient la science.

Qu'on se souvienne de ce que le rejet de la castration se soutient d'un " arrêt sur image »que pratique électivement le pervers avec ses montages fétichiques. ${ }^{6}$ La technologie de l'image organise méthodiquement cet "arrêt sur image ». Arrêt ciblé et savamment opérationnalisé.

Il ne s'agit pas de parler de perversion de la science, mais de nouer deux constats, l'un emprunté à la réalité même de la science venue sur le devant de la scène de ce vouloirvoir -, l'autre à la clinique analytique : là où ce " pousse-à-l'image " se notifie, on peut dépister un clivage sous-jacent. Plus " ça veut voir ", plus s'aggrave la fêlure entre l'œil et le regard. Plus l'image s'évertue à montrer, plus elle cache. L'écran même devient un "cache ». Et pour cause: la représentation se fonde d'un irreprésentable.

II y a donc bien avancée technique spectaculaire, sauf à s'aviser de son envers: la clinique de l'écoute se trouve virtuellement subvertie par un idéal de la monstration en son idéologie factualiste. À quoi bon parler, puisque l'essentiel est de montrer?

Quant au sujet, il est, comme l'indique le dispositif de l'examen IRM, littéralement placé dans un tunnel: devenu corps opaque plongé dans le noir (ce qui à l'occasion enclenche un vécu claustrophobique), comme s'il fallait payer de cette immersion dans l'opacité - véritable Ananké en forme de resserrement ${ }^{7}$ - les promesses d'une toute-transparence.

\section{Photographier le délire}

Ce mouvement atteint son paroxysme au moment où c'est l'organe cérébral qui est sommé de livrer la clé du symptôme. On comprend aussi que c'est sur la psychose qu'il achoppe.

On en arrive à vouloir voir comment le psychotique délire, c'est-à-dire ce qui se passe dans le corps, dans le creux du cerveau, quand il délire. Ce que l'on appelle "neuro-imagerie", ensemble de techniques permettant d'obtenir des images du cerveau par scanner et surtout imagerie par Résonance Magnétique (IRM), selon la technique tomographique indiquée. Là où le scanner utilise les rayons X, I'IRM analyse la concentration en hydrogène, caractéristique de chaque organe. L'image globale est reconstruite par ordinateur. Paradoxalement, le comble de l'image réelle est ici virtuelle. Tomographie par émission de positron, d'un seul photon. II s'agit dévaluer l'activité métabolique cérébrale et le débit sanguin.

L'IRM détecte les régions d'activité neuronale en enregistrant les changements des niveaux d'oxygénation sanguine. Lorsque l'activité neuronale augmente, le débit de sang oxygéné augmente aussi dans une région donnée. Excès dans la quantité de sang oxygéné par rapport au sang non oxygéné. Le signal de résonance magnétique vient signer l'activité fonctionnelle. Ce regard sur le symptôme psychotique se déroule donc dans une atmosphère objectivement surréaliste. Voilà un regard en aveugle sur les mouvements du délire. Tout du drame 
signifiant est gommé, non qu'il soit réfuté intellectuellement, mais mis hors jeu, sur cette base solide qu'il ne se voit pas. Car ce qui ne voit pas n'intéresse pas, étant in-concevable. C'est que, on le sait, l'imagier, lui, (ne) veut (que) voir. Si cette démarche folle - en quelque sorte homologue à la psychose- n'est pas sans fasciner, c'est qu'elle comble un vœu : celui de voir l'errance psychotique en direct plus encore qu'in statu nascendi, le délire live; mieux : de saisir le réel de dessous le délire.

Forclusion symbolique, dites-vous? C'est abstrait, nous, nous voulons voir comment jouit, où jouit le corps psychotique quand ça " débloque "! Pour la "physiognomonie » du XVIIle siècle qui voulait, tel Lavater, lire le caractère dans la structure du crâne, "l'esprit est un os ", disait Hegel: le délire, pour nos imagiers modernes, c'est une bouffée de chaleur cérébrale. Ainsi se profile le désir de l'imagier de prendre l'esprit du psychotique dans le bocal de l'image. Le réel psychotique veut être " touché des yeux ", appareillé par ces lunettes astronomiques du dedans. Cela n'est pas sans fasciner, dans la mesure où la psychose se soutient justement d'une délocalisation de la jouissance, corrélative de la forclusion symbolique. Mais l'on voit se dessiner l'ambition à terme, de forclore, au moyen de la neuroimagerie, la forclusion même.

On dira que l'ambition ne va pas si loin: pas question d'élucider la cause de la psychose, seulement le tracé physiologique précis de son déclenchement. Mais précisément ce n'est pas seulement de concomitants physiologiques du délire qu'il s'agit. Comme parallèlement le délire est coupé de toute théorie étio-pathogénique comme dans l'idéologie du catalogage du DSM -, l'imagerie en vient à définir le mode d'accès à l'événement, puis l'événement même.

Le fantasme froid de la science : I'avenir d'une illusion

Tel est le message ironique de la psychanalyse, loin de toute idéologie anti-moderniste ou misonéiste: la neuro-imagerie réalise la pratique d'une vision des plus directe et intrusive qui reconduit la division du sujet dans et par le dispositif fétichique de l'image. Elle contribue donc activement et concrètement, à son niveau d'un laboratoire de l'image, à la désanimation de la psychopathologie du sujet et du conflit. Inversion: là où la topologie du signifiant était, la topique de l'image advient.

Ce serait une illusion de chercher hors de la science ce qu'elle ne nous donne pas, avertit Freud en conclusion de son analyse de l'illusion en sa texture religieuse; mais voilà qu'elle reconduit l'illusion de l'image en gommant l'événement symbolique. C'est cette oblitération qui est à penser comme envers du donner à voir.

\section{L'œil artificiel ou le fantasme de la science}

Comment cela ne toucherait-il pas au fantasme ? ${ }^{8}$

Il s'agit, au-delà de l'objectif et légitime besoin de voir, du désir de visualiser l'intérieur - ce qui touche à la question du féminin. ${ }^{9}$ L'imagerie médicale a au reste une expansion spectaculaire dans le domaine gynécologique - l'exemple pouvant se confirmer ici comme "la chose même $"{ }^{10} \mathrm{Ne}$ s'agit-il pas de mettre l'intérieur à portée de la vision et en ligne de mire ?

Si cela relève du fantasme, c'est que précisément le regard butte sur un "irreprésentable » et que plus on " voit ", plus se trouve entourée l'opacité de la Chose. C'est au reste pourquoi il faut toujours voir de plus près, plus précisément, tenter de se placer à l'épicentre de ce quelque chose qui est invisible. C'est ce "pousse-auvoir » qui organise le voyeurisme tranquille de la science. Celui qui localise dans le Dedans ouvert "l'Origine du monde » qui chez Courbet donnait ses lignes à la transgression picturale, de déposséder implicitement le Père symbolique de cette fonction.

Corrélativement, le clivage tend à être institué, fût-ce au moyen d'une pensée strictement et rigoureusement " opératoire ». On assiste ainsi 
à une pétrification du fantasme. Bref, les techniques s'inscrivent dans une logique fétichique.

Le fétiche s'instaure, en sa dynamique inconsciente, au lieu même où se produit une répression du " manque », du " trou » d'origine. Il s'arrête au seuil de cet insupportable à voir qu'il s'agit de voiler, le "troumatisme » dont parle Lacan. C'est ce qui organise en retour une pulsion de voir quand même et coûte que coûte.

\section{La « schize » de l'œil et du regard}

Cela suppose un nouveau rapport entre œil et regard. Relisons de ce point de vue le diagnostic formulé par Kafka, faisant état d'une impression personnelle à propos du régime cinématographique de l'image :

Il est vrai que c'est un jouet magnifique. Mais je ne le supporte pas. Je suis un de ces êtres chez qui prime la vue. Or le cinéma perturbe la vision. La rapidité des mouvements et la succession précipitée des images vous condamnent à une vision superficielle de façon continue. Ce n'est pas le regard qui saisit les images, ce sont les images qui saisissent le regard. Elles submergent la conscience. Le cinéma contraint l'œil à endosser un uniforme, alors que jusqu'ici il était nu.

Voilà magnifiquement décrit le problème du rapport du regard à l'image. Kafka parle ici il est vrai du cinéma, et de plus en sa phase débutante de tâtonnement dans l'écriture iconographique. II n'en pointe pas moins une question structurelle. Il est à peine paradoxal de dire que la technique de l'image " perturbe " la vision, alors même qu'elle amplifie l'image et ouvre des espaces nouveaux à la vision.

L'imagerie médicale renvoie bien à un film, sauf à quêter le contraire du film, soit l'arrêt sur image. Il n'en aliène pas moins le regard clinique : I'œil n'est désormais plus « nu ». C'est là, il est vrai, l'avantage fondamental de la technique, prolongeant le grand mouvement entamé depuis le télescope et le microscope. Mais, précisément, du temps que l'œil était nu, il se posait sans cesse la question de la tension possible entre l'œil et le regard. C'est toujours au reste le statut irréductible du regard dans le Lebenswelt, ce monde de la vie hors de la science (et qui se révèle dans le "coup de foudre " amoureux). Le danger objectif est qu'une clinique de l'image institue la " schize » de l'œil et du regard.

Il est vrai qu'après tout la technique de l'image n'est ni plus ni moins qu'un instrument, destiné à fonder le jugement clinique et à l'informer. Mais précisément, ce faisant, le danger est qu'il lui donne forme, qu'il l'inscrive dans une "conformité de l'image» au moyen de ce "jouet magnifique " avec lequel le médecin joue et dont il sait de mieux en mieux jouer. Jouet dont le patient est l'usager... et lui-même le jouet, consentant ou non, consentant au nom de la science.

Si l'œil est la "fenêtre de l'âme ", les films et images pourraient être "les volets de fer " du regard.

L'autorité aveugle de l'image: le regard clinique

Cela n'est pas prétexte à suspicion misonéiste. Le regard fût-il serf de l'image, il ne peut en éviter désormais les fascinations et les pouvoirs. Reste, pour le regard clinique, à mesurer les conséquences de cette autorité aveugle de l'image. Aux promesses de guérison - ô combien précieuses-, le sujet sacrifie son regard.

La psychanalyse redécouvre le sujet comme "parlêtre ", se frayant la voie à son être à travers le double défilé de son image et du langage.

Il y a dans l'argument imparable de l'image quelque chose qui participe foncièrement de la dépossession de la parole - dès lors que le médecin abdique sa fonction d'écoute et de transfert ${ }^{11}$ pour se faire le "serveur" autant que le servant de l'image. Ce qui confirme le diagnostic freudien sur l'homme en sa fonction "prothétique " dans son lien au « malaise de la culture ». 
Il y aurait, pour paraphraser Freud, à dégager l'impératif que, là où était l'image, la parole doit advenir - non pour dénoncer quelque " malédiction » de l'image, mais en ce que, bien plutôt, en s' « édictant ", l'usage et le savoir de l'image fait sortir de la diction, se mettant illusoirement au-delà de l'alternative du « bien dire " ou du " mal dire " : illusion promise à un bel avenir, que le savoir de l'inconscient soumet obstinément à l'échéance de la vérité du sujet...

\section{Bibliographie :}

Assoun, P.-L. (1984). L'entendement freudien. Logos et Ananké. Paris : Gallimard.

Assoun, P.-L. (1993). Introduction à la métapsychologie freudienne. Paris : PUF.

Assoun, P.-L. (2004). Leçons de psychanalyse. Anthropos/Economica.

Assoun, P.-L. (2006). Le fétichisme. Paris : PUF.

Assoun, P.-L. (2007). Leçons psychanalytiques sur le fantasme. Anthropos/Economica.

Assoun, P.-L. (2007). Leçons psychanalytiques sur masculin et féminin. Anthropos/Economica.

Assoun, P.-L. (2007). Leçons psychanalytiques sur le transfert. Anthropos/Economica.

Freud, S. (1942). Trois essais sur la théorie sexuelle. Gesammelte Werke. Frankfurt-am-Main: S. Fischer Verlag. Freud, S. (1942). Pulsions et destins des pulsions. Gesammelte Werke. Frankfurt-am-Main: S. Fischer Verlag. Kafka, F. (1978). Conversations avec Gustav Janouch. Paris : Maurice Nadeau.

\section{Notes :}

${ }^{1}$ Kafka, Franz (1978). Conversations avec Gustav Janouch. Paris : Maurice Nadeau.

${ }^{2}$ Assoun, P.-L. (2004). Le regard et la voix. Leçons de psychanalyse. Anthropos/ Economica, $2^{\mathrm{e}}$ éd.

${ }^{3}$ Freud, S. (1942). Trois essais sur la théorie sexuelle. Gesammelte Werke. Frankfurt-am-Main: S. Fischer Verlag, t. V, 95.

${ }^{4}$ Freud, S. (1942). Pulsions et destins des pulsions. Gesammelte Werke. Frankfurt-am-Main: S. Fischer Verlag, t. X, 224.

${ }^{5}$ Assoun, P.-L.. Corps et symptôme. Leçons de psychanalyse, Op. cit., p. 119 sq.

${ }^{6}$ Assoun, P.-L. (2006). Le fétichisme. Paris : PUF, Que saisje ?, $3^{\mathrm{e}}$ éd.

${ }^{7}$ Assoun, P.-L. (1984). L'entendement freudien. Logos et Ananké. Paris : Gallimard.

${ }^{8}$ Assoun, P.-L. (2007). Leçons psychanalytiques sur le fantasme. Anthropos/Economica.

${ }^{9}$ Assoun, P.-L. (2007). Leçons psychanalytiques sur masculin et féminin. Anthropos/Economica, $2^{\mathrm{e}}$ éd.

${ }^{10}$ Assoun, P.-L. (1993). Introduction à la métapsychologie freudienne. Paris : PUF, "Quadrige ».

${ }^{11}$ Assoun, P.-L. (2007). Leçons psychanalytiques sur Le transfert. Anthropos/Economica, 2e éd.

\section{L'auteur :}

\section{Paul-Laurent Assoun}

Psychanalyste.

Professeur de Psychopathologie, Université

Paris VII Diderot

Campus Paris Rive Gauche

Bâtiment Olympe de Gouges

11, rue Jean Antoine de Baïf

75013 Paris

France

\section{Référence électronique}

Paul-Laurent Assoun, "L'image médicale à l'épreuve de la psychanalyse - Le fantasme iconographique ", Recherches en Psychanalyse [En ligne], 08|2009, mis en ligne le 12 décembre 2009.

Texte intégral

Droits d'auteur

Tous droits réservés 